\title{
Low miR-1231 expression predicts poor prognosis in non-small cell lung cancer and accelerates cell proliferation, migration and invasion
}

\section{Lina Zhu}

The Third Affiliated Hospital, Soochow University

\section{Chu Zhang}

The Third Affiliated Hospital, Soochow University

Hui Yu ( $\square$ neoplasm1216@163.com)

Affiliated Hospital of Jiangsu University

Lirong Zhu

Afffiliated Hospital of Jiangsu University

\section{Research}

Keywords: MiR-1231, Prognosis, Proliferation, Migration, Invasion, Non-small cell lung cancer

Posted Date: June 4th, 2020

DOI: https://doi.org/10.21203/rs.3.rs-32310/v1

License: (c) (i) This work is licensed under a Creative Commons Attribution 4.0 International License. Read Full License

Version of Record: A version of this preprint was published at Biomarkers in Medicine on August 1st, 2021. See the published version at https://doi.org/10.2217/bmm-2020-0313. 


\section{Abstract \\ Background}

MicroRNAs (miRNAs) have been confirmed to be involved in the tumor progression of various cancer types. This study aimed to assess the prognostic significance and biological function of miR-1231 in patients with non-small cell lung cancer (NSCLC).

\section{Methods}

The expression of miR-1231 was estimated using quantitative real-time polymerase chain reaction (qRT$\mathrm{PCR}$ ). Kaplan-Meier survival curves and Cox regression analysis were used to evaluate the prognosis value of miR-1231 in patients with NSCLC. Cell experiments were performed to assess the biological function of miR-1231 in the tumor progression.

\section{Results}

In this study, we found that miR-1231 was an important tumor suppressor with significantly low expression in NSCLC tissues and cell lines compared with normal controls (all $P<0.001$ ). MiR-1231 expression was significantly associated with tumor size $(P=0.037)$, lymph node metastasis $(P=0.001)$ and TNM stages $(P=0.001)$. Furthermore, the patients with low miR-1231 expression had shorter survival time than those with high miR-1231 expression (log-rank $P=0.010)$. In addition, miR-1231 was found to serve as an independent prognostic biomarker for the patients. The results of cell experiments indicated that miR-1231 downregulation could markedly promote NSCLC cell proliferation, migration and invasion, while miR-1231 overexpression could markedly inhibit NSCLC cell proliferation, migration and invasion.

\section{Conclusion}

All the data revealed that a downregulated expression of miR-1231 predicts the poor prognosis of NSCLC and promotes the tumor cell proliferation, migration and invasion. Therefore, we considered that miR1231 may serve as a therapeutic target for NSCLC treatment.

\section{Introduction}

Lung cancer has always been one of major malignancies worldwide, with an estimated 20.9 million new cases and 17.6 million people die from lung cancer in the GLOBOCAN database (1). Lung cancer includes both small cell lung cancer (SCLC) and non-small cell lung cancer (NSCLC), but the latter accounts for more than $80 \%$ of lung cancer cases (2). Lung cancer patients are usually diagnosed at advanced stages, and usually more than $60 \%$ of patients are at stage $\otimes$ or $\otimes$ before treatment (3).Thus, despite there is progress in cancer treatment, the prognosis of NSCLC remains poor and the 5-year survival rate of lung 
cancer is less than $20 \%$, the survival rate of this disease has remained virtually unchanged $(4,5)$. Therefore, improving the prognosis of NSCLC and exploring effective treatment methods are urgent problems that need to be solved clinically.

MicroRNAs (miRNAs) are a class of small endogenous non-coding RNAs with 19-25 nucleotides long (6), with regulatory role in gene expression by binding to the 3'-untranslated region (3'-UTR) of target mRNA (7). MiRNAs have been demonstrated to be involved in the regulation of oncogenes and tumor suppressors (8). Up to now, it has been well documented that alterations of miRNA expression contribute to the pathogenesis of most human cancers (9-11). A number of studies have explored that miRNA expression is also related to NSCLC (12-14) and miRNA has pro-cancer and anti-cancer effects is expressed differently in NSCLC (15). In addition, study has indicated that miR-1231 is a downstream target of LINC00673 overexpression in lung cancer tissues (16). And Zheng et al. (17) found that the alteration of exon no. 4 rs11655237 of LINC00673 creates a target site for the binding of miR-1231, thereby weakening the effect of LINC00673 in an allele-specific manner, resulting in susceptibility to tumorigenesis. Thus, the miR-1231 may also be involved in the progression of NSCLC.

In this study, we explored the expression level of miR-1231 in NSCLC tissues and cells, evaluated its prognostic significance in cancer patients, and finally discussed the effect of miR-1231 on the behavior of NSCLC cells. The results of this study may provide evidence for miR-1231 to serve as a potential biomarker and therapeutic target in the treatment of NSCLC.

\section{Materials And Methods}

\section{Patients and tissue collection}

A total of 121 NSCLC patients who had been subjected to resection surgery from the Third Affiliated Hospital, Soochow University were recruited between 2010 and 2014. All patients were pathologically diagnosed as NSCLC and none of patients had received any treatment in advance. Cancerous tissues and matched noncancerous tissues were collected from each patient and frozen with liquid nitrogen and store at $-80^{\circ} \mathrm{C}$ for further use. And all patients had complete electronic medical record information. The experimental procedure of this study was approved by the Ethics committee of the Third Affiliated Hospital, Soochow University, and each patient was required to sign a written informed consent. Each patient was followed up for 5 years and survival data were recorded.

\section{Cell culture and transfection}

Four NSCLC cell lines A549, H460, H1299 and HCC827, and a normal human lung epithelial cell line NHBE were obtained from the Central Culture Collection of the Chinese Academy of Science (Shanghai, China). The cells were cultured in Dulbecco's modified Eagle's medium (DMEM) supplemented with $10 \%$ fetal bovine serum (FBS, Gibco, CA, USA), and maintained at $37^{\circ} \mathrm{C}$ in a humidified incubator with $5 \% \mathrm{CO}_{2}$. After the incubation, the cells were transfected with miR-1231 mimic, miR-1231 inhibitor or their corresponding negative controls (mimic NC and inhibitor NC) using Lipofectamine ${ }^{\mathrm{TM}} 2000$ (Invitrogen) 
according to the manufacturer's protocols. The vectors used in the transfection were all obtained from GenePharma (Shanghai, China). Cells were collected after transfection for 48 hours and used for following analyses.

\section{RNA extraction and quantitative Real-time PCR (qRT-PCR)}

Total RNA was extracted from the tissues and cells by TRIzol reagent (Invitrogen) according to the instructions of the manufacturers. The collected RNA was quantified by NanoDrop 2000 (Thermo Fisher Scientific).

MiR-1231 expression was examined using qRT-PCR, which was carried out with SYBR Green I PCR Core Reagent (Applied Biosystems, Foster City, CA) with a 7500 Real-Time PCR system (Applied Biosystems). U6 was used as an internal control, and the final expression data of miR-1231 was calculated using the $2^{-\triangle \Delta C t}$ method.

\section{Cell proliferation assay}

After transfection, the stably transfected cells were grown in complete medium in 96-well plates with $5 \times$ $10^{3}$ cells/well. Each well was added to $20 \mu \mathrm{L}$ MTT (Sigma-Aldrich, MO, USA) after incubated for 24,48 and $72 \mathrm{~h}$, and was further cultured for $4 \mathrm{~h}$ at $37^{\circ} \mathrm{C}$. The medium was then removed and $150 \mu \mathrm{L}$ of dimethyl sulfoxide (DMSO) was added to the wells to dissolve the formazan. Finally, absorbance at $490 \mathrm{~nm}$ was detected to evaluate the cell proliferation by a microplate reader (Bio-Rad, model 550, Philadelphia, PA, USA).

\section{Cell migration and invasion assay}

Transwell system with $8 \mu \mathrm{m}$ pore size (Coring, USA) were used to analyze the migration and invasion abilities of the NSCLC cells. The system was divided into two types: one without matrigel precoating (for migration assay) and the other with matrigel coating (for invasion assay). The same composition in the upper and low chambers was the DMEM, and the difference is that the low chamber was filled with $10 \%$ FBS. The tumor cells were inoculated into the upper chamber. After $48 \mathrm{~h}$ incubation at $37^{\circ} \mathrm{C}$, the cells in the bottom chambers were stained and counted using an inverted microscope. The experiments were repeated at least three times.

\section{Statistical analysis}

All data were presented as the mean \pm SD and all statistical analyses were performed using SPSS 21.0 software (SPSS, Inc., Chicago, USA) and GraphPad Prism 7.0 software (Inc., Chicago, USA). The data of two group or multiple groups were compared using Student's t-test or ANOVA followed by Tukey's test. Relationship between miR-1231 expression and clinicopathological data was analyzed using Chi-square test. Survival analysis was performed using Kaplan-Meier methods and log-rank test. Prognostic value of miR-1231 was confirmed by Cox regression analysis. Differences with $P<0.050$ were considered statistically significant. 


\section{Results}

\section{Downregulation of miR-1231 in NSCLC tissues and cell lines}

By the qRT-PCR, we observed that the miR-1231 expression was significantly downregulated in NSCLC tissues compared with the normal controls $(P<0.001$, Fig. 1A). Similarly, the expression of miR-1231 in all four NSCLC cell lines were also downregulated compared with the normal cells (all $P<0.010$, Fig. 1B).

\section{Association of miR-1231expression with clinicopathological characteristics of NSCLC patients}

The role of miR-1231 in the tumor development of NSCLC was examined by investigating the relationship between miR-1231 and the clinicopathological data of cancer patients. According to the median value of miR-1231 expression, the NSCLC patients were grouped into low $(n=64)$ and high $(n=57)$ miR-1231 expression groups. From the data in Table 1, the expression of miR-1231 was found to be associated with tumor size $(P=0.037)$, lymph node metastasis (LNM, $P=0.001)$ and TNM stage $(P=0.001)$, whereas no significant relationship was found between miR-1231 and age, gender or smoking (all $P>0.050$ ). 
Table 1

Association of miR-1231 expression and the clinical characteristics of NSCLC patients

\begin{tabular}{|c|c|c|c|c|}
\hline \multirow[t]{2}{*}{ Features } & \multirow{2}{*}{$\begin{array}{l}\text { Total No. } \\
n=121\end{array}$} & \multicolumn{2}{|c|}{ miR-1231 expression } & \multirow[t]{2}{*}{$P$ values } \\
\hline & & $\operatorname{Low}(n=64)$ & High $(n=57)$ & \\
\hline Age (Years) & & & & 0.958 \\
\hline$\leq 60$ & 47 & 25 & 22 & \\
\hline$>60$ & 74 & 39 & 35 & \\
\hline Gender & & & & 0.783 \\
\hline Female & 44 & 24 & 20 & \\
\hline Male & 77 & 40 & 37 & \\
\hline Tumor size $(\mathrm{cm})$ & & & & 0.037 \\
\hline$\leq 3$ & 60 & 26 & 34 & \\
\hline$>3$ & 61 & 38 & 23 & \\
\hline Smoking & & & & 0.777 \\
\hline No & 43 & 22 & 21 & \\
\hline Yes & 78 & 42 & 36 & \\
\hline Lymph node metastasis & & & & 0.001 \\
\hline Negative & 65 & 25 & 40 & \\
\hline Positive & 56 & 39 & 17 & \\
\hline TNM stage & & & & 0.001 \\
\hline$I-\nabla$ & 60 & 23 & 37 & \\
\hline $\mathbb{Q}-\mathbb{Z}$ & 61 & 41 & 20 & \\
\hline
\end{tabular}

Because of the dysregulation in the expression of miR-1231 in NSCLC clinical samples, this test further investigated the clinical significance of miR-1231 in the prognosis of NSCLC. The Kaplan-Meier survival curves (Fig. 2) indicated that patients with low expression levels of miR-1231 had clearly shorter survival time than those with high expression levels of miR-1231 (log-rank $P=0.010)$. Furthermore, our multivariate Cox analysis showed that miR-1231 ( $\mathrm{HR}=2.024,95 \% \mathrm{Cl}=1.158-3.537, P=0.013)$ and TNM stage $(\mathrm{HR}=1.808,95 \% \mathrm{Cl}=1.081-3.024, P=0.024)$ were two independent prognostic factors for the survival in patients with NSCLC (Table 2). 
Table 2

Multivariate Cox analysis for miR-1231 in NSCLC patients

\begin{tabular}{|llll|}
\hline Variables & \multicolumn{3}{l|}{ Multivariate analysis } \\
\cline { 2 - 4 } & $\mathrm{HR}$ & $\mathbf{9 5 \%} \mathrm{Cl}$ & $\boldsymbol{P}$ value \\
\hline miR-1231 & 2.024 & $1.158-3.537$ & 0.013 \\
\hline Age & 1.063 & $0.637-1.774$ & 0.816 \\
\hline Gender & 1.050 & $0.634-1.741$ & 0.849 \\
\hline Tumor size & 1.341 & $0.830-2.167$ & 0.231 \\
\hline Smoking & 1.192 & $0.711-1.997$ & 0.505 \\
\hline Lymph node metastasis & 1.416 & $0.866-2.316$ & 0.166 \\
\hline TNM stage & 1.808 & $1.081-3.024$ & 0.024 \\
\hline
\end{tabular}

\section{MiR-1231 promotes NSCLC cell proliferation}

To further understand the function of miR-1231 in NSCLC progression, A549 and H1299 were selected for cell experiments as they had dramatically low expression of miR-1231. By cell transfection, the expression of miR-1231 in the two cell lines was all upregulated by the miR-1231 mimic, whereas was downregulated by the miR-1231 inhibitor (all $P<0.010$, Fig. 3A and B). By using MTT assay, we found that the overexpression of miR-1231 suppressed, but the downregulation of miR-1231 promoted the cell proliferation (all $P<0.010$, Fig. 3C and D) in both A549 and H1299 cells.

\section{Effect of miR-1231 on cell migration and invasion of NSCLC cells}

In addition to cell proliferation, the effect of miR-1231 on migration and invasion were also examined in the A549 and H1299 cells by Transwell assay. As presented in Fig. 4A and 4B, the migration of cancer cells was promoted by the downregulation of miR-1231, but was suppressed by the upregulation of miR1231 compared with the untreated cells (all $P<0.050$ ). Similarly, the invasion of cancer cells was also promoted by miR-1231 reduction, whereas was suppressed by miR-1231 overexpression (all $P<0.010$, Fig. 4C and 4D)

\section{Discussion}

MiRNAs are a kind of endogenous non-coding single-stranded small RNAs that are key regulators of gene expression and are involved in almost all tumor-related processes $(9,18)$. Because the miRNAs effect the post transcriptional expression of different genes, they are currently the focus of cancer studies (19). By different upregulation or downregulation and affecting the expression of genes involved in cancer cell signaling, miRNAs regulate cancer cells proliferation, invasion and apoptosis (20). Therefore, miRNAs are 
divided into two kinds: oncogenic miRNAs and suppressive miRNAs (20). For example, Cai et al. (21) found that miR-519a is a suppressive miRNA in gastric cancer and the downregulation of miR-519a predicts poor prognosis. While the study of Jian et al. (22) indicated that miR-193-3p functions as an oncogenic miRNA in gastric cancer progression. All these studies showed that the functional miRNAs could be used as novel therapeutic targets for cancer targeted therapy.

Increasing evidence have documented that miRNAs play vital roles in NSCLC progression by regulating NSCLC cell biological function. For example, overexpression of miR-24 could significantly enhanced NSCLC cell migration and invasion by inhibiting ZNF367 (23). MiR-451a inhibits the migration and invasion of NSCLC cells by accelerating AFT2 (5). MiR-135a inhibited proliferation, migration and invasion in NSCLC cells through targeting IGF-1 gene (24). And miR-185 inhibited the proliferation, migration and invasion of NSCLC cells by targeting KLF7 (25). Our study found that the expression level of miR-1231 was downregulated in NSCLC tissues and cell lines, which led us to further explore the relationship between miR-1231 and NSCLC.

In this study, we found that the expression level of miR-1231 was significantly lower in NSCLC controls compared with normal tissues. Similarly, the miR-1231 expression in NSCLC cell lines was significantly lower than those normal cells. It also indicated that the expression level of miR-1231 in NSCLC was downregulated. Additionally, most of the patients with low miR-1231 expression showed lymph node metastasis and presented with an advanced TNM stage. Thus, it is assumed that miR-1231 may be associated with the development of NSCLC. Moreover, the miR-1231 expression was also altered in other types of human cancers. MiR-1231 plays a tumor-suppressive role through regulating the EGFR/PI3K/AKT AXIS in glioma and was an independent prognosis factor for glioma $(26,27)$. MiR-1231 expression was downregulated in prostate cancer and may suppress prostate cancer cell proliferation, migration and invasion by targeting EGFR (28). Exosomal miR-1231 inhibit the expression of pancreatic cancer $(29,30)$. The upregulated miR-1231 was found in colorectal cancer, which may be related to the onset of colorectal cancer (31). Therefore, we predicts that miR-1231 may act as a suppressive miRNA in NSCLC.

By analyzing the 5-year survival information, we also explored the prognosis value of miR-1231 in NSCLC patients. The relationship of miR-1231 with survival outcomes has been analyzed in some cancers, such as pancreatic cancer, glioma and prostate cancer (26-29). However, as far as we know, the clinical significance of miR-1231 in NSCLC has rarely been reported. In our study, we plotted the Kaplan-Meier survival curves of NSCLC patients based on the recorded 5-year survival information. The curves showed that patients with low miR-1231 expression had shorter survival time than those patients with high miR1231 expression. Additionally, the Cox regression data indicated that the expression of miR-1231 was an independent prognosis factor. Overall, the decreased miR-1231 may predict the poor prognosis of NSCLC.

To further explore the biological function of miR-1231 in NSCLC progression, we used the miR-1231 mimic and miR-1231 inhibitor to perform cell experiments. After cell transfection, the miR-1231 expression was promoted by the miR-1231 mimic and was reduced by the miR-1231 inhibitor. The 
upregulation of miR-1231 could inhibit, whereas the downregulation of miR-1231 could enhance the NSCLC proliferation, migration and invasion, indicating that miR-1231 acted as a tumor suppressor in NSCLC. Previous studies have reported that miR-1231 plays a tumor-suppressive role through regulating the EGFR/PI3K/AKT signaling in glioma $(26,27)$. However, whether this signaling pathway also mediated the regulatory effect of miR-1231 on NSCLC progression remains unclear, and further investigations are needed to further uncover the molecular mechanisms underlying the role of miR-1231 in NSCLC.

\section{Conclusion}

In summary, this study indicated that the expression level of miR-1231 is reduced in NSCLC and serves as an independent prognosis biomarker. The upregulation of miR-1231 was indicated to suppress NSCLC cell proliferation, migration and invasion, which prompts that miR-1231 may function as a therapeutic target in the treatment of NSCLC.

\section{Declarations}

\section{Ethics approval and consent to participate}

A signed written informed consent was obtained from each patient and the experimental procedures were all in accordance with the guideline of the Ethics Committee of the Third Affiliated Hospital, Soochow University.

\section{Consent for publication}

Written informed consent for publication was obtained from each participant.

\section{Availability of data and material}

All data generated or analyzed during this study are included in this published article.

\section{Conflicts of interest}

The authors have declared no conflict of interest.

\section{Funding}

None.

\section{Authors' contributions}

L. N. Zhu carried out the design and conception of the research. L. N. Zhu and C. Zhang analyzed and interpreted the data regarding, H. Yu and L. R. Zhu performed the examination of cell, and wrote and revised the manuscript. L. N. Zhu revised the manuscript critically for important intellectual content. Both authors read and approved the final manuscript. 
Acknowledgements

Not applicable.

\section{References}

1. Ferlay J, Colombet M, Soerjomataram I, Mathers C, Parkin DM, Pineros M, et al. Estimating the global cancer incidence and mortality in 2018: GLOBOCAN sources and methods. International journal of cancer. 2019;144(8):1941-53.

2. Gyoba J, Shan S, Roa W, Bedard EL. Diagnosing Lung Cancers through Examination of Micro-RNA Biomarkers in Blood, Plasma, Serum and Sputum: A Review and Summary of Current Literature. Int J Mol Sci. 2016;17(4):494.

3. Wu KL, Tsai YM, Lien CT, Kuo PL, Hung AJ. The Roles of MicroRNA in Lung Cancer. International journal of molecular sciences. 2019;20(7).

4. Siegel RL, Miller KD, Jemal A. Cancer statistics. 2016. CA: a cancer journal for clinicians. 2016;66(1):7-30.

5. Shen YY, Cui JY, Yuan J, Wang X. MiR-451a suppressed cell migration and invasion in non-small cell lung cancer through targeting ATF2. Eur Rev Med Pharmacol Sci. 2018;22(17):5554-61.

6. Cui R. Linking miR-138 and USP10-P53 signaling. Oncotarget. 2019;10(61):6541-2.

7. Vishnoi A, Rani S. MiRNA Biogenesis and Regulation of Diseases: An Overview. Methods in molecular biology. 2017;1509:1-10.

8. Hrovatin $\mathrm{K}$, Kunej T. Classification of heterogeneous genetic variations of microRNA regulome in cancer. Cancer letters. 2018;419:128-38.

9. Fang RH, Ji XB. [Advances in the research of the relationship between miRNA-29c and cancer]. Lin chuang er bi yan hou tou jing wai ke za zhi = Journal of clinical otorhinolaryngology, head, and neck surgery. 2018;32(4):312-7.

10. Rupaimoole R, Slack FJ. MicroRNA therapeutics: towards a new era for the management of cancer and other diseases. Nature reviews Drug discovery. 2017;16(3):203-22.

11. Javanmardi S, Aghamaali MR, Abolmaali SS, Mohammadi S, Tamaddon AM. miR-21, An Oncogenic Target miRNA for Cancer Therapy: Molecular Mechanisms and Recent Advancements in Chemo and Radio-resistance. Current gene therapy. 2017;16(6):375-89.

12. Lu J, Zhan Y, Feng J, Luo J, Fan S. MicroRNAs associated with therapy of non-small cell lung cancer. Int J Biol Sci. 2018;14(4):390-7.

13. Mizuno K, Mataki H, Seki N, Kumamoto T, Kamikawaji K, Inoue H. MicroRNAs in non-small cell lung cancer and idiopathic pulmonary fibrosis. Journal of human genetics. 2017;62(1):57-65.

14. Chen YJ, Guo YN, Shi K, Huang HM, Huang SP, Xu WQ, et al. Down-regulation of microRNA-144-3p and its clinical value in non-small cell lung cancer: a comprehensive analysis based on microarray, miRNA-sequencing, and quantitative real-time PCR data. Respiratory research. 2019;20(1):48. 
15. Yerukala Sathipati S, Ho SY. Identifying the miRNA signature associated with survival time in patients with lung adenocarcinoma using miRNA expression profiles. Scientific reports. 2017;7(1):7507.

16. Qiao F, Li N, Li W. Integrative Bioinformatics Analysis Reveals Potential Long Non-Coding RNA Biomarkers and Analysis of Function in Non-Smoking Females with Lung Cancer. Medical science monitor: international medical journal of experimental clinical research. 2018;24:5771-8.

17. Zheng J, Huang X, Tan W, Yu D, Du Z, Chang J, et al. Pancreatic cancer risk variant in LINC00673 creates a miR-1231 binding site and interferes with PTPN11 degradation. Nat Genet. 2016;48(7):747-57.

18. Fiammengo R. Can nanotechnology improve cancer diagnosis through miRNA detection? Biomark Med. 2017;11(1):69-86.

19. Masood N, Basharat Z, Khan T, Yasmin A. Entangling Relation of Micro RNA-let7, miRNA-200 and miRNA-125 with Various Cancers. Pathology oncology research: POR. 2017;23(4):707-15.

20. Qadir MI, Faheem A. miRNA:. A Diagnostic and Therapeutic Tool for Pancreatic Cancer. Crit Rev Eukaryot Gene Expr. 2017;27(3):197-204.

21. Cai H, Lin H, Cao W, Sun J, Huang Y, Fang Y. The downregulation of miR-519a predicts poor prognosis and contributes to tumor progression in gastric cancer. Int $\mathrm{J}$ Clin Exp Pathol. 2019;12(7):2496-505.

22. Jian B, Li Z, Xiao D, He G, Bai L, Yang Q. Downregulation of microRNA-193-3p inhibits tumor proliferation migration and chemoresistance in human gastric cancer by regulating PTEN gene. Tumour biology: the journal of the International Society for Oncodevelopmental Biology Medicine. 2016;37(7):8941-9.

23. Liu Z, Jiang L, Zhang G, Li S, Jiang X. MiR-24 promotes migration and invasion of non-small cell lung cancer by targeting ZNF367. Journal of BUON: official journal of the Balkan Union of Oncology. 2018;23(5):1413-9.

24. Zhou Y, Li S, Li J, Wang D, Li Q. Effect of microRNA-135a on Cell Proliferation, Migration, Invasion, Apoptosis and Tumor Angiogenesis Through the IGF-1/PI3K/Akt Signaling Pathway in Non-Small Cell Lung Cancer. Cellular physiology and biochemistry: international journal of experimental cellular physiology, biochemistry, and pharmacology. 2017;42(4):1431-46.

25. Zhao L, Zhang Y, Liu J, Yin W, Jin D, Wang D, et al. miR-185 Inhibits the Proliferation and Invasion of Non-Small Cell Lung Cancer by Targeting KLF7. Oncology research. 2019;27(9):1015-23.

26. Wang H, Wu J, Luo WJ, Hu JL. Low expression of miR-1231 in patients with glioma and its prognostic significance. Eur Rev Med Pharmacol Sci. 2018;22(23):8399-405.

27. Zhang J, Zhang J, Qiu W, Zhang J, Li Y, Kong E, et al. MicroRNA-1231 exerts a tumor suppressor role through regulating the EGFR/PI3K/AKT axis in glioma. Journal of neuro-oncology. 2018;139(3):54762.

28. Wang Y, Zhang Q, Guo B, Feng J, Zhao D. miR-1231 Is Downregulated in Prostate Cancer with Prognostic and Functional Implications. Oncology research treatment. 2020;43(3):78-86. 
29. Chen SL, Ma M, Yan L, Xiong SH, Liu Z, Li S, et al. [Clinical significance of exosomal miR-1231 in pancreatic cancer]. Zhonghua zhong liu za zhi [Chinese journal of oncology]. 2019;41(1):46-9.

30. Shang S, Wang J, Chen S, Tian R, Zeng H, Wang L, et al. Exosomal miRNA-1231 derived from bone marrow mesenchymal stem cells inhibits the activity of pancreatic cancer. Cancer medicine. 2019;8(18):7728-40.

31. Tan YG, Zhang YF, Guo CJ, Yang M, Chen MY. Screening of differentially expressed microRNA in ulcerative colitis related colorectal cancer. Asian Pacific journal of tropical medicine. 2013;6(12):972-6.

\section{Figures}

A

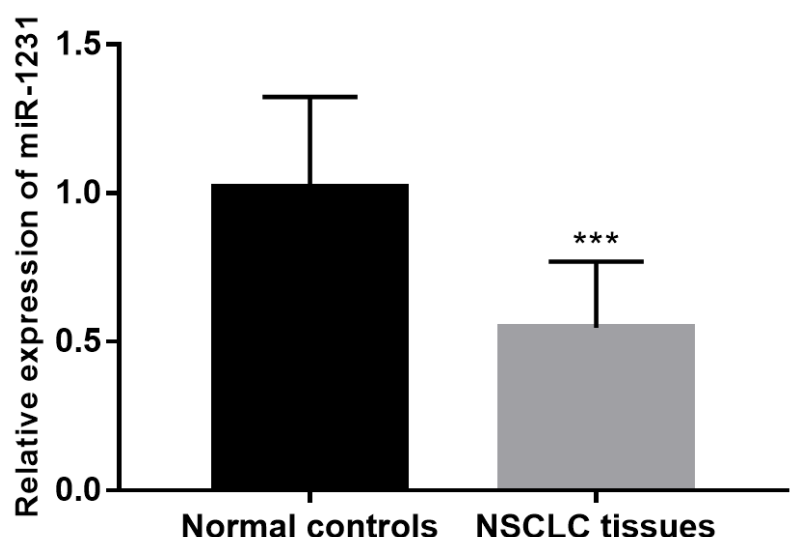

B

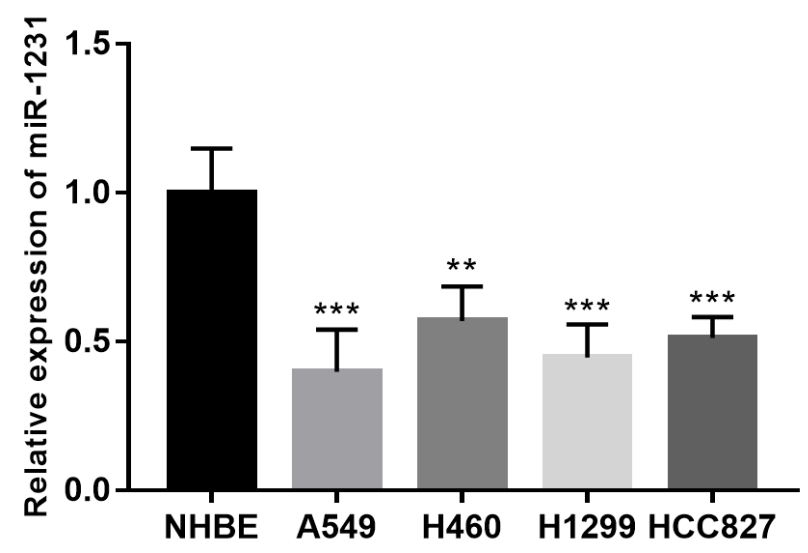

\section{Figure 1}

Expression of miR-1231 in NSCLC tissues and cell lines. (A) The expression of miR-1231 was downregulated in NSCLC tissues compared with the normal controls. (B) Lower expression level of miR1231 was founded in four NSCLC cell lines compared with the normal cells. ${ }^{\star *} \mathrm{P}<0.010,{ }^{\star \star *} \mathrm{P}<0.001$. 


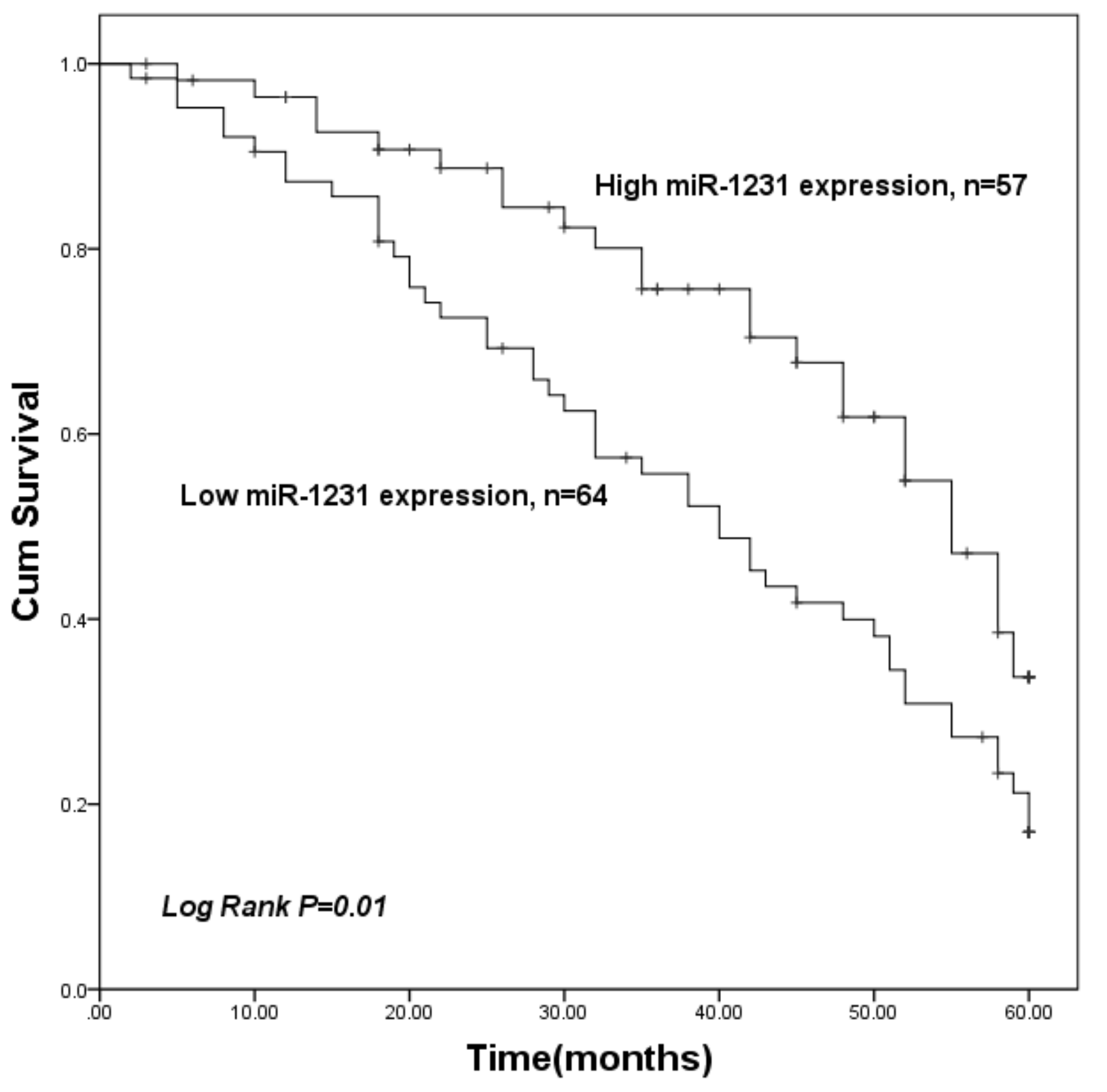

Figure 2

Kaplan-Meier survival curves in the patients with NSCLC. The survival time of patients with low miR-1231 expression was shorter than those patients with high miR-1231 expression. (Log-Rank P $=0.010$ ) 
A

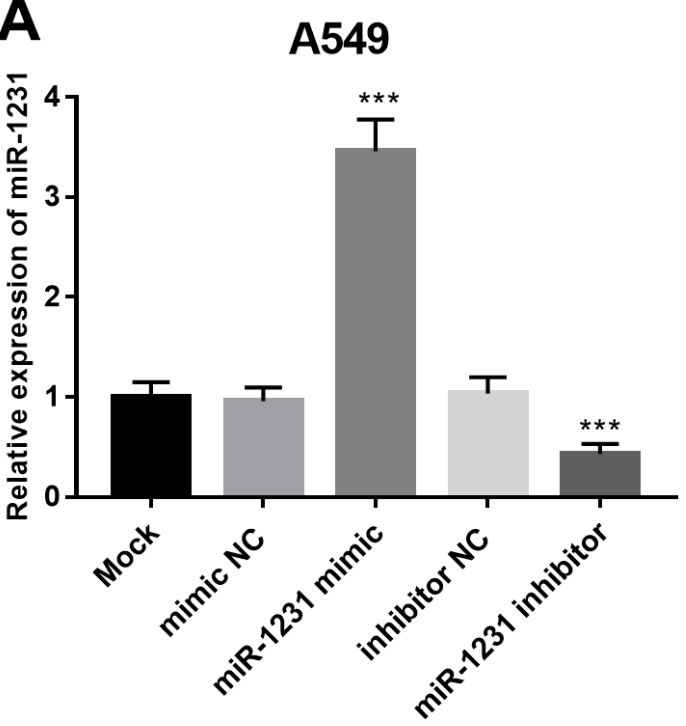

C

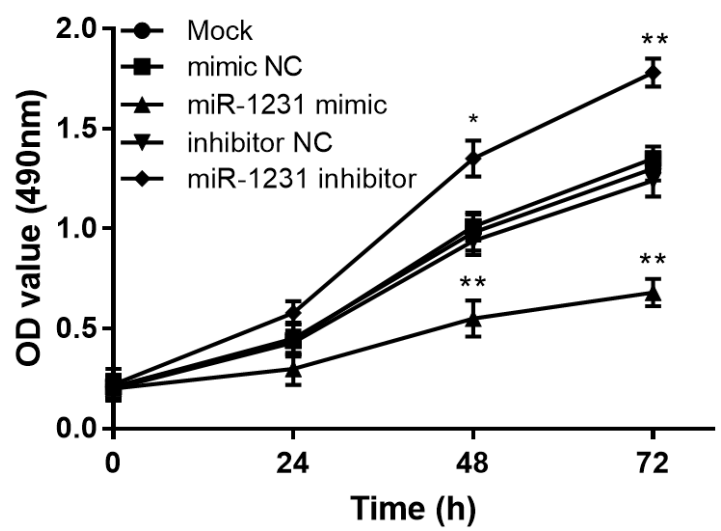

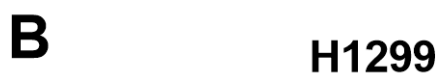

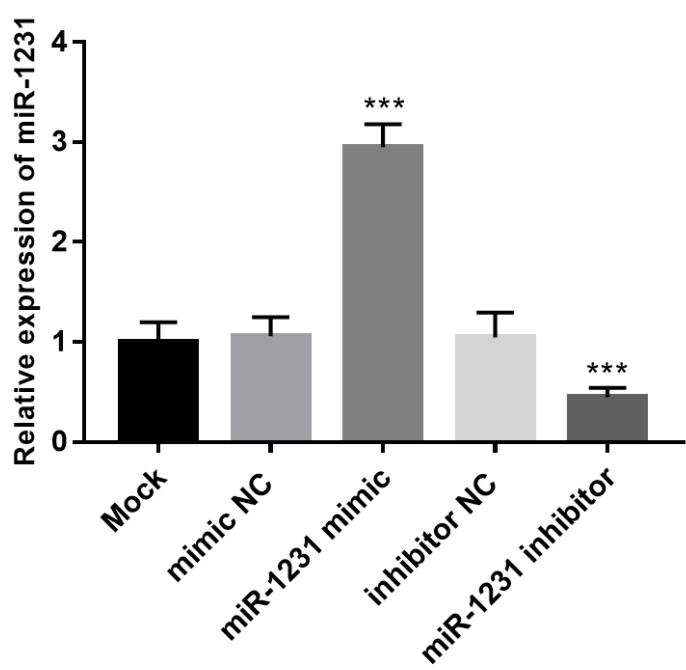

D

H1299

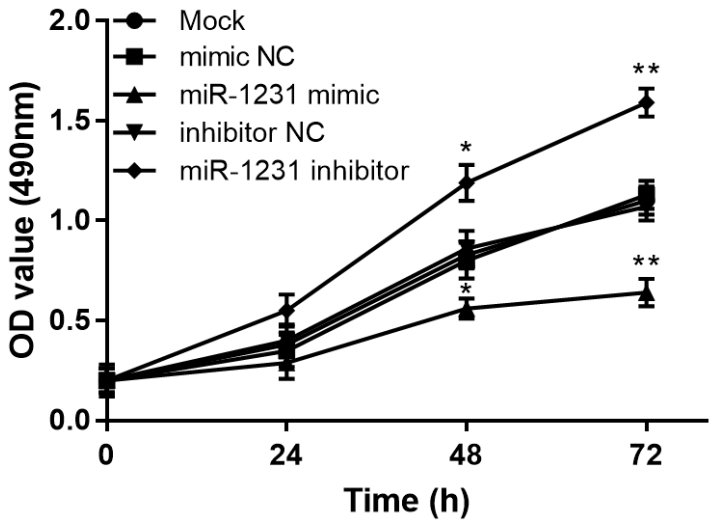

Figure 3

Effect of miR-1231 on proliferation of A549 and H1299 cells. The expression of miR-1231 in (A) A549 and (B) H1299 cells was significantly higher following transfection with miR-1231 mimic and significantly lower following transfection with miR-1231 inhibitor compared with untreated cells. The proliferation of (C) A549 and (D) H1299 cells was inhibited when the expression of miR-1231 was increased, and was enhanced when the expression of miR-1231 was decreased. ${ }^{*} P<0.050, * \star P<0.010$, $\star \star \star P<0.001$. 
A

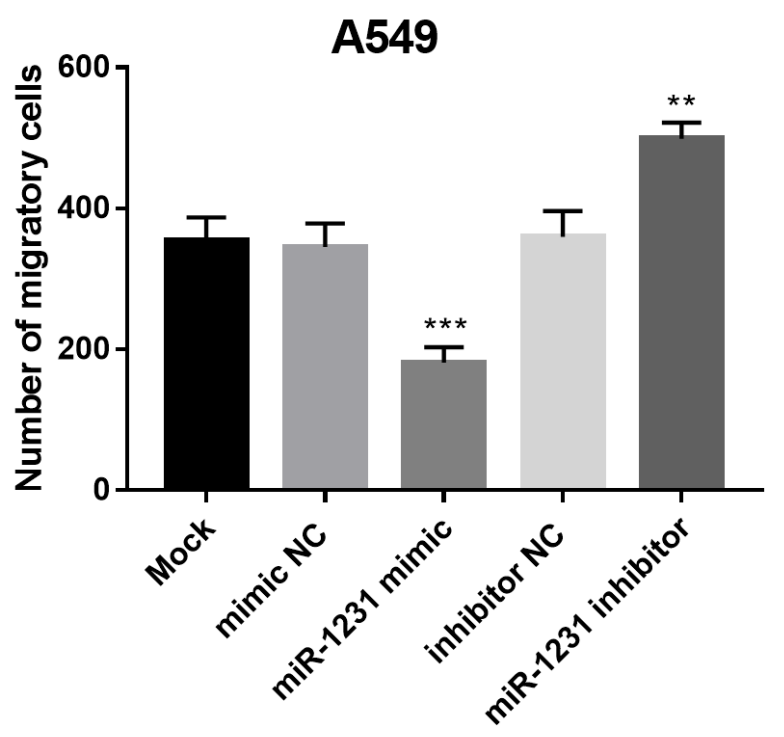

C

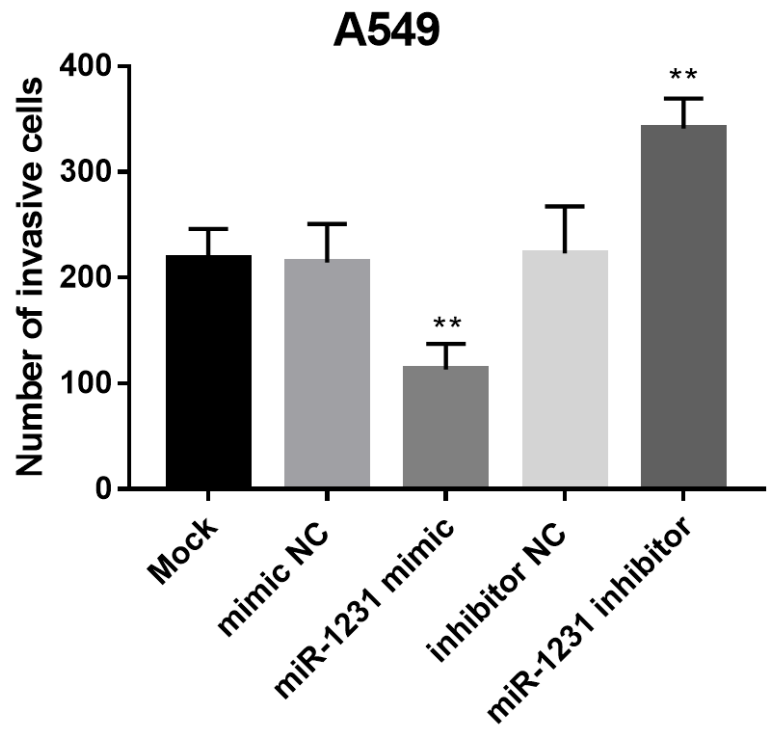

B

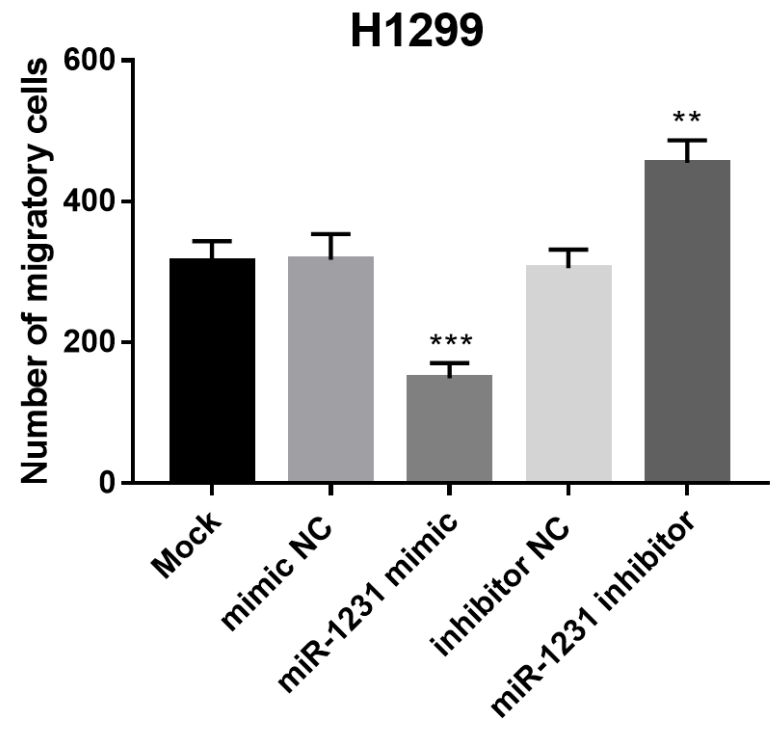

D

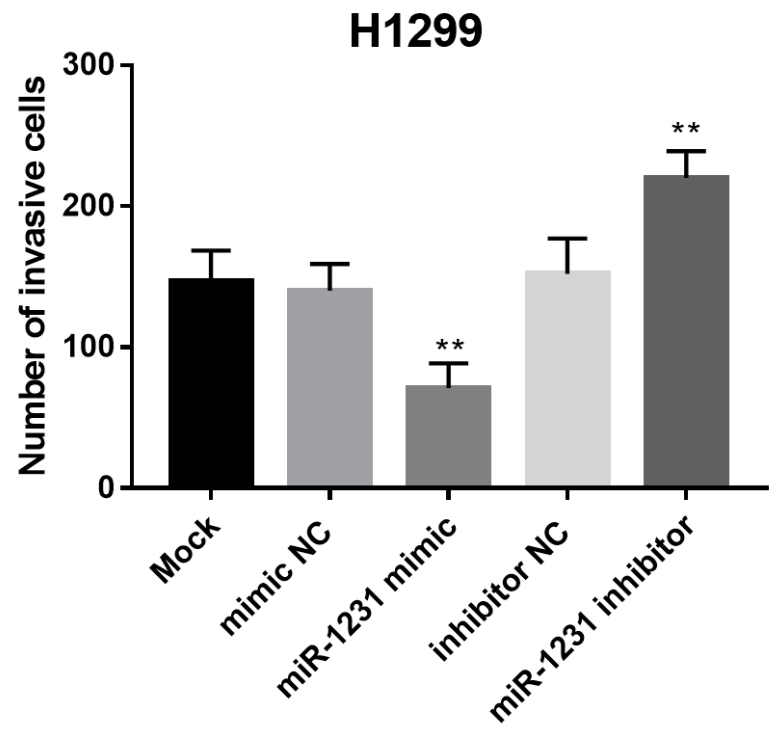

Figure 4

Effect of miR-1231 on the migration and invasion of A549 and H1299 cells. The migration of (A) A549 and (B) H1299 cells was inhibited by the upregulation of miR-1231, but was enhanced by downregulation of miR-1231. Similarly, miR-1231 overexpression inhibited, whereas miR-1231 downregulation promoted the invasion of (C) A549 and (D) H1299 cells. All experiments were repeated three times. ${ }^{*} \mathrm{P}<0.010$, ${ }^{\star * *} \mathrm{P}$ $<0.001$. 